\title{
MicroRNA-223 Suppresses Osteoblast Differentiation by Inhibiting DHRS3
}

\author{
Shijie Zhang ${ }^{a, b} \quad$ Yi Liu ${ }^{b} \quad$ Zhong Zheng ${ }^{c, d} \quad$ Xuemin Zeng ${ }^{b} \quad$ Dongxu Liub \\ Chunling Wang ${ }^{b}$ Kang Ting ${ }^{c, d}$
}

aDepartment of Orthodontics, Qilu Hospital of Shandong University, Jinan, Shandong bShandong Provincial Key Laboratory of Oral Tissue Regeneration, School of Stomatology, Shandong University \& Department of Orthodontics, School of Stomatology, Shandong University, Jinan, Shandong, China; 'Section of Orthodontics, Division of Growth and Development, School of Dentistry, University of California, Los Angeles, CA dDivision of Plastic and Reconstructive Surgery and Department of Orthopaedic Surgery, the Orthopaedic Hospital Research Center, University of California, Los Angeles, CA, USA

\section{Key Words}

miR-223 • DHRS3 • BMSC • Osteogenic differentiation

\begin{abstract}
Background/Aims: In this study, we aimed to use bioinformatics tools to identify the specific miRNAs and mRNAs involved in osteogenic differentiation and to further explore the way in which miRNA regulates osteogenic differentiation. Methods: The microarray GSE80614, which includes data from 3 human mesenchymal stromal cells (hMSCs) and 3 hMSCs after 72 hours (hr) of osteogenic differentiation, was used to screen for differentially expressed mRNAs. Gene ontology (GO) and Kyoto Encyclopedia of Genes and Genomes (KEGG) pathway analyses of these mRNAs were conducted using Gene Set Enrichment Analysis (GSEA). Then, the miRanda website was employed to detect the binding sites of DHRS3. In vitro experiments, including RT-PCR and western blotting, were used to detect miR-233 and DHRS3 expression levels 7 and 14 days (d) after the induction of osteogenic differentiation using human bone marrow-derived mesenchymal stem cells (hBMSCs). The target relationship between miR-223 and DHRS3 was confirmed by a dual luciferase assay. ALP (alkaline phosphatase) staining, ARS (Alizarin Red S) staining and western blotting (Runx2, OPN, OCN) were used to detect the level of osteogenic differentiation after transfection with miR-223 mimics and DHRS3 CDNA. Results: In this study, 127 mRNAs differentially expressed during osteogenic differentiation were identified in GSE80614. GO term and KEGG pathway enrichment analyses found that the retinol metabolism pathway was activated during osteogenic differentiation and that DHRS3, which is involved in the pathway, was upregulated. During osteogenic differentiation in hBMSCs, miR-223 was gradually downregulated, while DHRS3 was upregulated. After 14 days of osteogenic differentiation, ALP and ARS staining assay results showed strong ALP activity and extracellular matrix calcification with the inhibition of miR-223 or the overexpression
\end{abstract}


of DHRS3. Furthermore, the expression levels of Runx2, OPN, and OCN were upregulated with the knockdown of miR-223 or the overexpression of DHRS3, while the simultaneous transfection of a miR-223 agomir and DHRS3 cDNA resulted in no significant difference from the negative control (NC) group. Conclusion: The inhibition of miR-223 promotes the osteogenic differentiation of hBMSCs via the upregulation of DHRS3.

(C) 2018 The Author(s)

Published by S. Karger AG, Basel

\section{Introduction}

It has been widely reported that mesenchymal stem cells (MSCs) play important roles in tissue engineering and cell therapies $[1,2]$. For example, exposure to a specific environmental stimulus, can induce MSCs to differentiate into varieties of adult tissues, such as bone, cartilage, fat and muscle $[3,4]$. The human bone marrow-derived mesenchymal stem cells (hBMSCs), a type of pluripotent stem cells, were the first members of the mesenchymal stem cell (MSC) family to be identified [5]. As the progenitor cells of osteoblasts, hBMSCs play a critical role in bone formation and differentiation, becoming an excellent source of seed cells in bone tissue engineering $[2,6,7]$.

Human quality of life may be seriously affected by bone defects caused by congenital malformations, traumas or diseases, imposing large economic and social burdens [8]. To treat or prevent these diseases, the key issue is promoting osteogenesis. A study by Wei et al. showed that in mouse models, the inhibition of osteoblast differentiation significantly reduced bone loss [9]. Thus, osteogenic differentiation is a key factor in bone regeneration, and clarifying the regulatory mechanism of osteoblast differentiation is very important for the improvement of the treatment of bone-related diseases.

MicroRNAs (miRNAs), a type of small noncoding RNAs, are able to bind to the 3'-untranslated regions (3'-UTRs) of specific target mRNAs, thereby regulating gene expression [10]. Studies have shown that miR-223 participates in the regulation of cardiomyocyte glucose metabolism, myoblast proliferation and differentiation, esophageal squamous cell carcinoma and human granulopoiesis [11-13]. In the osteogenic differentiation of hBMSCs, miR-223 was found to act as both a positive and a negative regulator, suggesting its potential in the treatment of bone-related diseases [14]. It was reported that the level of miR-223 is significantly lower in osteosarcoma tissues compared with non-cancerous bone tissues [15]. Moreover, the expression of miR-223 is significantly higher in the synovium of patients with osteoarthritis than in the synovium of healthy individuals [16]. In another study by Xie et al., miR-223 was found to play a role during osteoblast differentiation [17]. However, the detailed mechanism of action of miR-223 during the osteogenic differentiation of hBMSCs has only been partially explored.

Retinol (vitamin A) is a micronutrient of vital importance in cell proliferation and differentiation. In adults, retinol and metabolites such as retinoic acid play key roles in vision, tissue remodeling and metabolism, as well as immune and brain functions. For instance, in the pituitary, retinoid might be helpful for the treatment of corticotropinomas by targeting the corticotroph; in the thyroid, vitamin A deficiency aggravates iodine-deficiency-related thyroid dysfunction [18]. In addition, retinoic acid, the metabolite of retinol, can cooperate with BMP-2 to enhance osteoblast differentiation through the induction of the expression of alkaline phosphatase $(A L P)$, osteocalcin $(O C N)$, and osteopontin $(O P N)$ [19]. It was also reported that RA promotes osteogenic differentiation in osteosarcoma cells, which is mediated in part by activating the Smad signaling pathway [20]. In all relevant studies, to the best of our knowledge, the effect of retinol metabolism during osteogenic differentiation has not yet been comprehensively identified.

The retinoic acid-inducible dehydrogenase reductase 3 (DHRS3) is a highly conserved member of the short-chain alcohol dehydrogenase/reductase superfamily and has been implicated in retinol metabolism [21]. Acting as a retinaldehyde reductase, DHRS3 is thought to control the levels of retinaldehyde, the immediate precursor of bioactive retinoic acid. Reversible oxidation-reduction reactions occur between retinol and retinaldehyde that are 


\section{Cellular Physiology Cell Physiol Biochem 2018;47:667-679

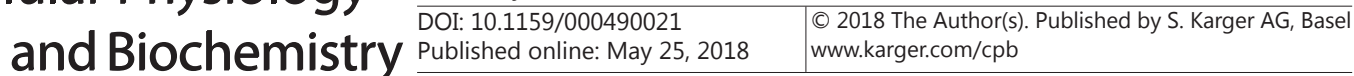 \\ Zhang et al.: MiR-223/DHRS3 and Osteoblast Differentiation}

regulated by RDH10/DHRS3 complex. Retinaldehyde can be further oxidized to RA [22], which may be involved in the regulation of osteogenic differentiation, as mentioned above. DHRS3 plays a critical role in regulating the metabolism of RA. The expression of DHRS3 is upregulated when the amount of RA is excessive and downregulated when RA synthesis or signaling is blocked [23]. In mice, mutation of DHRS3 leads to excess RA in embryos [24]. Although there are numerous studies of the effects of DHRS3 on retinol metabolism, information about the effects of DHRS3 on osteogenic differentiation is still very sparse, and the exact mechanism of action of DHRS3 remains unclear. The present study aimed to resolve this issue.

In the present study, we screened mRNAs that were differentially expressed during osteogenic differentiation and analyzed the key pathways and involved genes by using gene set enrichment analysis. Activation of the retinol metabolism pathway and overexpression of DHRS3 were found during the differentiation of osteoblasts. MiR-223 was found to target DHRS3 and was downregulated during osteoblast differentiation. This study was undertaken to clarify how miR-223 regulates osteogenic differentiation by affecting the retinol metabolism pathway and DHRS3.

\section{Materials and Methods}

\section{Cell culture and transfection}

The hBMSC cell lines and the human embryonic kidney cell line 293T were all purchased from the BeNa Culture Collection (BNCC339828, Beijing, China). The cells were all maintained in high-glucose Dulbecco's modified Eagle's medium (DMEM) with 10\% fetal bovine serum (FBS), $4 \mathrm{mM}$ L-glutamine (Gln) and sodium pyruvate and incubated in a $5 \% \mathrm{CO}_{2}$ atmosphere at $37^{\circ} \mathrm{C}$. The miR-223 agomir, the miR-223 antagomir and their negative controls, generated by GenePharma (Shanghai, China), were transfected into hBMSCs according to the manufacturer's instruction. The transfection was performed with Lipofectamine 2000 reagent according to the manufacturer's instructions. Transfection efficiency was detected, and the cells were used for further experiments.

\section{Gene expression profiles}

The gene expression data matrix was obtained from the National Center for Biotechnology Information Gene Expression Omnibus (GEO) (https://www.ncbi.nlm.nih.gov/geo/), which is accessible through the GEO platform GPL6947 and Series Accession Number GSE80614. The matrix contained a total of 66 samples, including osteogenic and adipogenic differentiation groups at different points in time ( 0 hour (hr), $0.5 \mathrm{hr}, 1$ hr, 2 hr, 3 hr, 6 hr, 12 hr, 1 day (d), 2 d, 3 d and 4 d), with each time point consisting of 3 cases. In the present study, we selected 3 samples after 0 hr of osteogenic differentiation as the normal group and 3 samples after 4 days of osteogenic differentiation as the osteogenic group.

\section{Screening differentially expressed mRNAs}

R software (Version 3.3.3) and Bioconductor packages (impute and limma) were applied to analyze the differentially expressed genes (DEGs) between the osteogenic differentiation samples and the normal samples. First, quality detection on microarray data was conducted by box plot, and quantile normalization was used to eliminate parallel experimental error. A clustering analysis diagram was constructed based on the "impute" and "limma" packages. Second, "ImFit" was chosen to process the data. The empirical Bayes method was employed to search for the significant DEGs. The $P$ values were adjusted by the BenjaminiHochberg procedure, and both adjusted $P<0.05$ and $\log _{2}$ (fold change (FC) $>2$ were considered statistically significant.

DAVID analysis and gene set enrichment analysis (GSEA)

Gene Ontology (GO) and Kyoto Encyclopedia of Genes and Genomes (KEGG) analyses were applied to investigate the underlying functions and some vital pathways involved in osteogenic differentiation. The DAVID Bioinformatics Tool (https://david.ncifcrf.gov/) was used for the GO and KEGG analyses. Three categories were exported from the GO analysis, namely, biological process (BP), cellular component (CC) 


\section{Cellular Physiology Cell Physiol Biochem 2018;47:667-679 \begin{tabular}{l|l} 
and Biochemistry Published 10.1159/000490021 2018 & $\begin{array}{l}\text { (c) } 2018 \text { The Author(s). Published by S. Karger AG, Basel } \\
\text { www.karger.com/cpb }\end{array}$ \\
\hline
\end{tabular} \\ Zhang et al.: MiR-223/DHRS3 and Osteoblast Differentiation}

and molecular function (MF). The $P$ value denotes the significance of the GO term enrichment among differentially expressed genes $(P<0.05)$. Pathway analysis was used to map the genes to KEGG pathways. The $P$ value denotes the significance of the pathway correlations $(P<0.05)$.

Gene set enrichment analysis (GSEA) was conducted based on the KEGG database. The expression data of total normalized mRNAs were uploaded to the GSEA v3.0 software. The default weighted enrichment statistic was adopted to process the data 1000 times, with normalized $P<0.05$ considered to be significantly enriched. Next, 7 of the highest up- and down-regulated results of the GSEA reports were selected to undergo graphics processing using the "ggplot2" package in R. Dotplot and ridgeplot were also used to visualize the data from the GSEA and illustrate the distribution of multiple pathways.

\section{Correlation network analysis of DEGs}

The expression profiles of genes involved in the KEGG_Retinol_Metabolism were analyzed through GSE80614. The differentially expressed genes with $\log _{2}$ (fold change (FC)) $>2$ and adjusted $P<0.05$ were selected and analyzed by hierarchical clustering. Meanwhile, TargetScan (http://www.targetscan.org/) and miRanda (microRNA.org) were applied to predict the microRNA targeting DHRS3, ADH1B, and ADH1A. Then, the networks were graphed using Cytoscape software.

\section{Osteoblast differentiation induction}

To induce osteogenic differentiation, hBMSC cells were grown to 80\% 90\% confluence, and then the medium was changed to osteoblast-specific induction medium, $\alpha$-MEM supplemented with 7.5\% FBS, 10 mM glycerol-2-phosphate (Sigma-Aldrich, St. Louis, MO, USA), $20 \mathrm{mM}$ L-ascorbic acid (Sigma-Aldrich), and $0.1 \mathrm{mM}$ dexamethasone (Sigma-Aldrich). The culture medium was replaced every 3 days for a period of 14 days. The cells were harvested and analyzed at 0,7 and 14 days.

\section{Quantitative Real-time PCR (qRT-PCR)}

TRIzol reagent (Thermo Fisher Scientific, Waltham, MA, USA) was used to extract the total RNA, and the iScript ${ }^{\mathrm{TM}}$ cDNA Synthesis Kit (Biorad, China) was used to synthesize cDNA following the manufacturer's protocol. iQ ${ }^{\text {TM }}$ SYBR Green Supermix (Biorad, China) was used to perform qRT-PCR with a 7500 HT Fast RealTime PCR System (Applied Biosystems). The relative gene expression levels of miR-223 and DHRS3 were evaluated using U6 and GAPDH as the endogenous normalization controls. The primer sequences of the evaluated genes are listed in Table 1.

\section{Western Blotting}

Whole cell lysates for western blotting were extracted with lysis buffer, and the total protein concentration was determined with a protein assay kit (Beyotime, Shanghai, China) following the manufacturer's instructions. Total protein $(20 \mu \mathrm{g})$ was boiled for $5 \mathrm{~min}$ in $1 \times$ loading buffer, chilled on ice, and then separated on $10 \%$ sulfate sodium salt-polyacrylamide gel electrophoresis (SDS-PAGE) and transferred to a polyvinylidene fluoride (PVDF) membrane (Merck Millipore, Billerica, MA, USA). The membranes were then blocked by incubation with $5 \%$ fat-free milk in TBST buffer $(50 \mathrm{mM}$ Tris- $\mathrm{HCl}, 150 \mathrm{mM} \mathrm{NaCl}, 0.05 \%$ Tween $20, \mathrm{pH} 7.6$ ) at $4^{\circ} \mathrm{C}$ for $1 \mathrm{~h}$. The membranes were incubated overnight with primary antibody for DHRS3

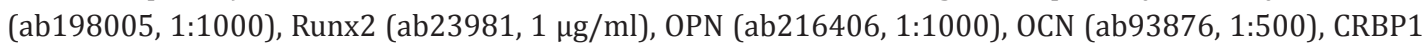
(ab154881, 1:1000), $\beta$-catenin (ab6302, 1:4000), and GAPDH (ab9485, 1:2500) at $4^{\circ} \mathrm{C}$. Unbound antibody was removed by washing the membranes in TBST buffer three times (10 min/wash). The membranes were then incubated with secondary antibody (ab6721, 1:5000) for $1 \mathrm{~h}$ at room temperature, and washed with TBST buffer three times (10 min/wash). The proteins were detected with an enhanced chemiluminescence detection system (Amersham Biosciences), followed by exposure to X-ray films.

Dual Luciferase Reporter Assay

The segments of DHRS33'UTR-WT (wild type) or DHRS33'UTR-MUT (mutant) were
Table 1. Specific primer sequences for qRT-PCR

\begin{tabular}{|c|c|c|}
\hline & Forward primers & Reverse primers \\
\hline DHRS3 & 5'-СTCTTTTACCGTCCСAACCTT-3' & 5'-GGGCATCATCATCACTGTCCA-3' \\
\hline miR-223 & 5'-CGTTGTCAGTTTGTCAAATAC-3' & 5'-CAGTGCAGGGTCCGAGGT-3' \\
\hline U6 & 5'-CTCGCTTCGGCAGCACA-3' & 5'-AACGCTTCACGAATTTGCGT-3' \\
\hline GAPDH & 5'-GTTCGACAGTCAGCCGCATC-3' & 5'-GGAATTTGCCATGGGTGGA-3' \\
\hline
\end{tabular}




\section{Cellular Physiology Cell Physiol Biochem 2018;47:667-679

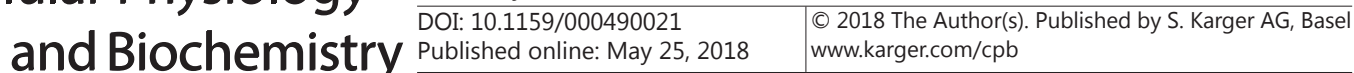 \\ Zhang et al.: MiR-223/DHRS3 and Osteoblast Differentiation}

constructed and then inserted into the luciferase reporter assay vector pmirGLO (Promega, Madison, USA). The vectors were transfected into 293T cells in accordance with the manufacturer's protocols. The cells were harvested $48 \mathrm{~h}$ after transfection with miR-223 mimics and miR-NC (GenePharma, Shanghai, China), and the luciferase activity was measured using the Dual Luciferase Reporter Assay System (Promega, WI, USA).

Alkaline phosphatase staining (ALP) and alizarin red staining (ARS)

Fourteen days after the induction of osteogenic differentiation, the BCIP/NBT Alkaline Phosphatase Color Development kit (Beyotime, China) was used to perform ALP staining according to the manufacturer's instructions. The Alkaline Phosphatase Assay kit (Nanjing Jiancheng Bioengineering Institute, China) was used to perform quantitative ALP measurements.

ARS was performed at $14 \mathrm{~d}$ to detect osteoblast calcification. Cells were washed twice with PBS, fixed in $95 \%$ ethanol for $10 \mathrm{~min}$, washed with distilled water three times, and then stained by Alizarin Red S staining solution (Cyagen, China), with the $\mathrm{pH}$ adjusted to 8.3 by $\mathrm{HCl}$, for $30 \mathrm{~min}$ at $37^{\circ} \mathrm{C}$. After being rinsed twice with distilled water, the cells were photographed.

\section{Statistical Analysis}

All experiments were repeated at least three times. The data were analyzed using Graph Pad Prism 6.0 software. All data are presented as the mean \pm standard deviation (SD). Differences between two groups were identified by unpaired $t$-tests, and one-way analysis of variance (ANOVA) was used to identify differences between three or more groups. $P<0.05$ was considered statistically significant.

\section{Consent for publication}

Consent for publication was obtained from the participants.

\section{Results}

Differentially expressed mRNAs during osteogenic differentiation and enrichment analysis

Expression levels were considered different if they were under the standard $\log _{2}(\mathrm{FC})>2$ and adjusted $P<0.05$. In total, 87 upregulated mRNAs and 40 downregulated mRNAs were identified (Table 2). Fig. 1 A shows the top $10 \mathrm{mRNAs}$ that are upand downregulated during osteogenic differentiation. Using DAVID for the KEGG pathway and GO enrichment analyses, we identified several vital pathways involved in osteogenic differentiation. The results showed that the differentially expressed mRNAs were significantly enriched in 8 pathways $(P<0.05$, Fig. 1B), including the AMPK signaling pathway and retinol metabolism. Fig. $1 \mathrm{C}-\mathrm{E}$ shows the 8 significantly upregulated GO terms in the biological process, cellular component and molecular function categories. According to the enrichment score from the GSEA report, the top 7 scored pathways

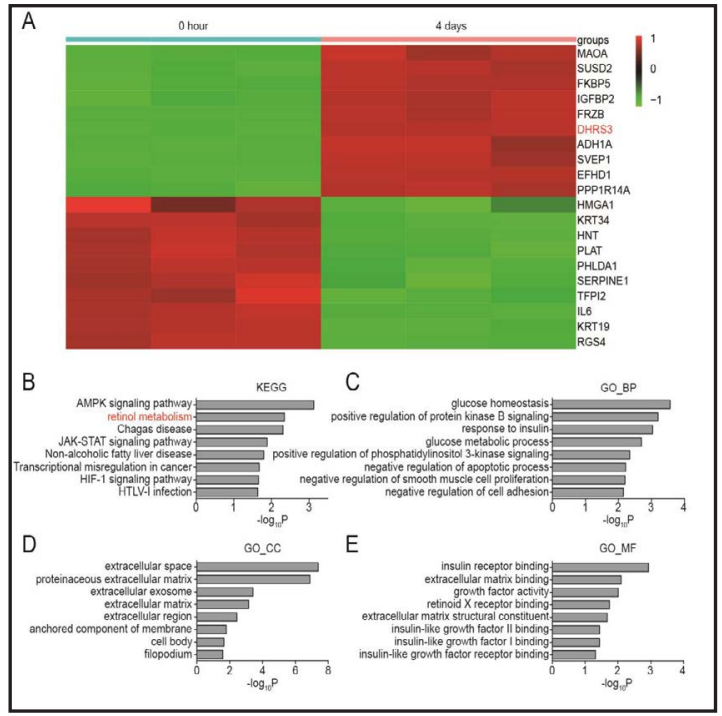

Fig. 1. Differentially expressed mRNAs during osteogenic differentiation. (A) The top 10 up- and downregulated mRNAs in normal and osteogenic differentiation samples were arranged by unsupervised hierarchical clustering. Data displayed as red and green represent elevated (high) and reduced expression (low), respectively. (B) Enrichment scores for Kyoto Encyclopedia of Genes and Genomes (KEGG) pathways that were the most significantly upregulated pathways during osteogenic differentiation. (CE) Enrichment scores of Gene Ontology (GO) terms that were upregulated most significantly, covering the domains of biological processes (BP), cellular components (CC) and molecular functions (MF). 


\begin{tabular}{|c|c|c|}
\hline \multirow[t]{2}{*}{ Cellu } & \multicolumn{2}{|c|}{ Cell Physiol Biochem 2018;47:667-679 } \\
\hline & $\begin{array}{l}\text { DOI: 10.1159/000490021 } \\
\text { Published online: : May 25, } 2018\end{array}$ & $\begin{array}{l}\text { O } 2018 \text { The Author(s). Published by S. Karger AG, Basel } \\
\text { www.karger.com/cpb }\end{array}$ \\
\hline
\end{tabular}

Table 2. Microarray GSE80614 analysis results. 87 upregulated mRNAs and 40 downregulated mRNAs were identified under the standard $\log _{2}(\mathrm{FC})>2$ and adjusted $\mathrm{P}<0.05$

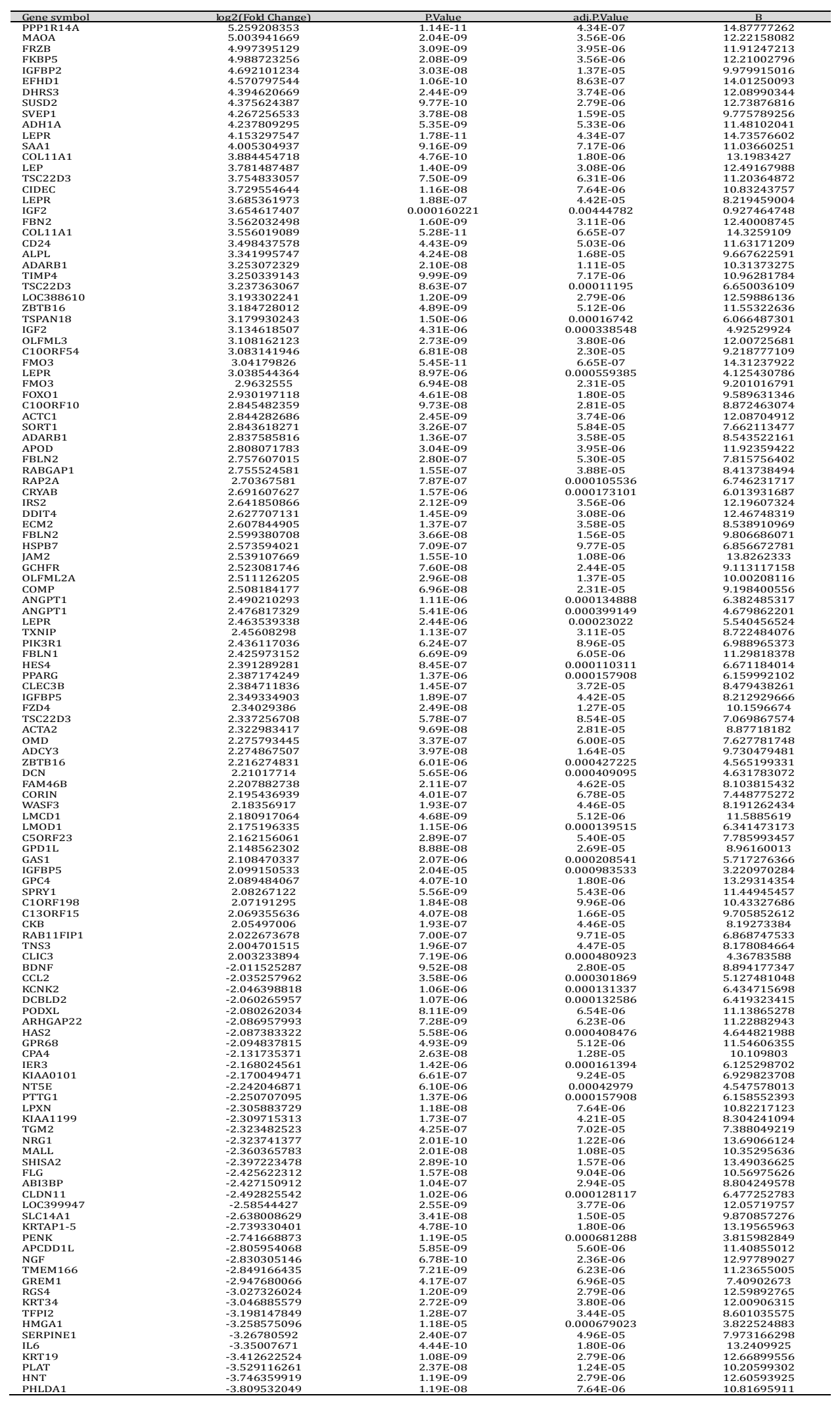


involved in osteogenic differentiation were ranked and shown in Fig. 2A. The enrichment of these pathways is shown in Fig. 2B using Cytoscape software. Based on the adjusted $P$-value of each pathway, the distributions of several KEGG pathways were drawn in dotplot (Fig. 3A) and ridgeplot (Fig. 3B). Both dotplot and ridgeplot showed that retinol metabolism was one of the most significantly activated pathways during osteogenic differentiation. Combining these bioinformatics results, we speculated that retinol metabolism could influence gene functions, thereby affecting osteogenic differentiation.

\section{Retinol metabolism during osteogenic differentiation}

The GSEA enrichment plot showed that most genes involved in retinol metabolism were found in the region where genes were upregulated during

Fig. 3. Distributions of several KEGG pathways as shown by dotplot and ridgeplot. (A) The distributions of some KEGG pathways gene sets during osteogenic differentiation. The retinol metabolism pathway was upregulated. In the dotplot, the enrichment significance (the adjusted $P$ value) is reflected by the color intensity of the nodes. The node size represents the gene count in the analyzed gene set. The gene ratio on the horizontal axis represents the proportion of differentially expressed genes in the gene set. (B) The distributions of some KEGG pathways gene sets during osteogenic differentiation. The retinol metabolism pathway gene sets were mainly located in the upregulated region. In the ridgeplot, the enrichment significance (the adjusted $P$ value) is reflected by the color intensity of the peaks. The value on the horizontal axis is the logarithm of the fold change. Peaks to the left of 0 indicate downregulation, while those to the right of 0 indicate upregulation.

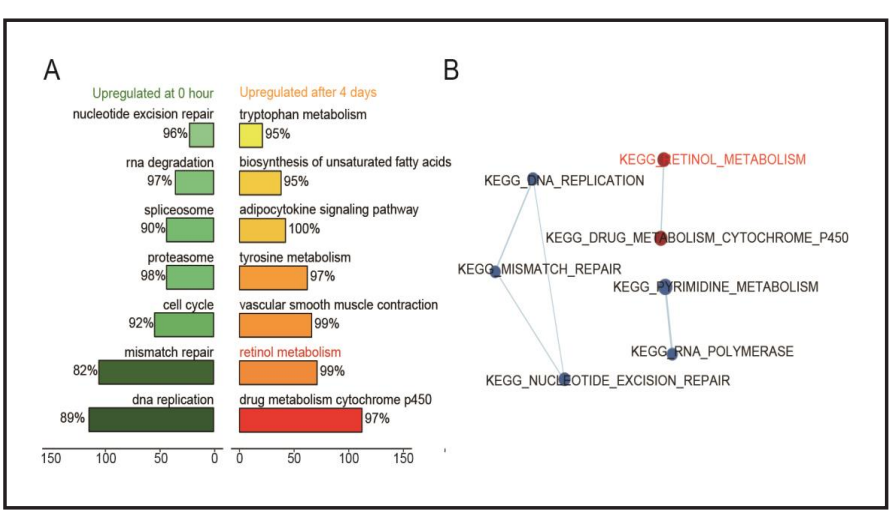

Fig. 2. Enrichment maps of KEGG pathways during osteogenic differentiation. (A) The 7 most distinctively activated pathways in the normal and osteogenic differentiation samples. The retinol metabolism pathway ranked the sixth in the most highly upregulated pathways during osteogenic differentiation. (B) Two pathways were identified as upregulated pathways, and 5 pathways were downregulated.

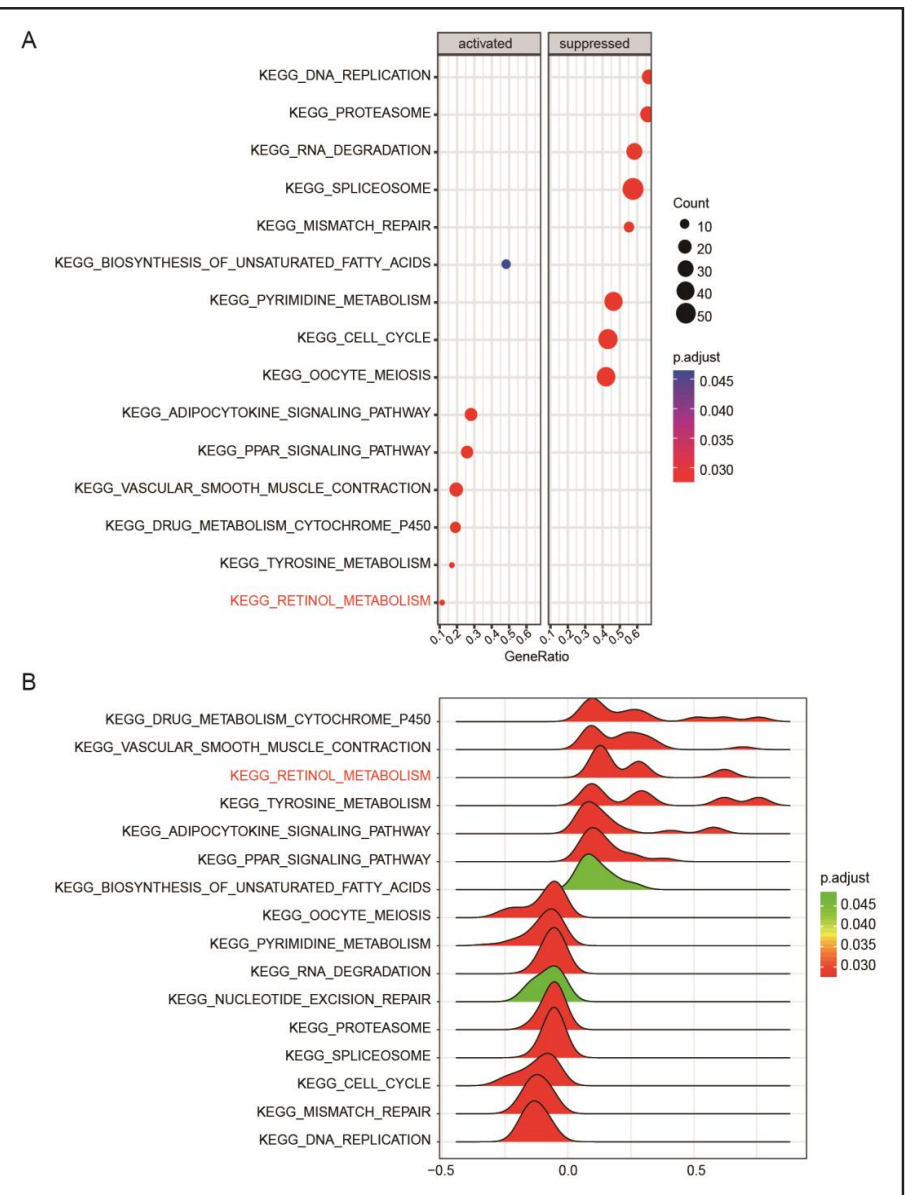




\section{Cellular Physiology Cell Physiol Biochem 2018;47:667-679 \begin{tabular}{l|l} 
DOI: 10.1159/000490021 & O 2018 The Author(s). Published by S. Karger AG, Basel \\
www.karger.com/cpb
\end{tabular}

osteogenic differentiation

(Fig. 4A). Therefore, retinol metabolism was activated in osteogenic differentiation. Among the genes thatareinvolved in retinol metabolism, the 3 genes with the most significantly upregulated expression were selected (Fig. 4B), namely, DHRS3, $A D H 1 A$, and $A D H 1 B$. Then, miRanda and TargetScan were used to screen for the microRNAs targeting those three genes (Fig. 4C). According to a previous study, miR223 plays an important role during osteoblast differentiation [17]. Thus, in the present study, we aimed to further investigate the mechanism by which miR223 affects osteogenic differentiation. Moreover, because $A D H 1 A$ and $A D H 1 B$ are two dehydrogenases, we further investigated the roles of miR-223 and DHRS3. The binding sites of miR-223 and DHRS3 are shown in Fig. 4D.

MiR-223 had a low expression level, while DHRS3 was highly expressed during the osteogenic differentiation of hBMSCs

The expression levels of miR-223 and DHRS3 after the osteogenic differentiation of hBMSCs were detected by qRT-PCR and western blotting. As shown in Fig. 5A-B, the expression of miR-224 gradually decreased while the expression of DHRS3 gradually increased during osteogenic differentiation (from $0 \mathrm{~d}$ to $14 \mathrm{~d}$ ). The western blotting results showed the same results; the expression of DHRS3 was increased $7 \mathrm{~d}$ and $14 \mathrm{~d}$ after the induction of osteogenic differentiation (Fig. 5C, $P<0.01$ ), which was consistent with the microarray analysis results.

\section{MiR-223 targeted the 3'UTR region of DHRS3}

As mentioned above, the prediction by TargetScan and miRanda suggested that DHRS3 might be the target of miR-223. The luciferase reporter gene assay was performed to verify their binding relationship. The result revealed that the luciferase activity of cells cotransfected with miR-223 mimics and 3'UTR-WT was significantly lower than that of cells co-transfected with NC and 3'UTR-WT (Fig. 6A, $P<0.01$ ), whereas miR-223 mimics had no effect on the fluorescence of the MUT recombinant plasmid (Fig. 6A, $P>0.05$ ). Moreover, we found that miR-223 could not target Runx2, OCN, or OPN (data not shown). Taken together, these results revealed that miR-223 directly targeted the 3'UTR of DHRS3.

MiR-223 prevented the osteogenic differentiation of hBMSCs by co-regulating retinol metabolism via suppressing DHRS3 expression

The results of qRT-PCR showed that miR-223 expression was upregulated in the miR223 agomir group, while it was downregulated in the miR-223 antagomir group, indicating 


\section{Cellular Physiology Cell Physiol Biochem 2018;47:667-679 \begin{tabular}{l|l} 
DOI: 10.1159/000490021 & O 2018 The Author(s). Published by S. Karger AG, Basel \\
www.karger.com/cpb
\end{tabular} \\ Zhang et al.: MiR-223/DHRS3 and Osteoblast Differentiation}

Fig. 5. Expression levels of miR-223 and DHRS3 changed during osteogenic differentiation. (A-B) QRT-PCR results detected that miR-223 expression was downregulated while DHRS3 mRNA expression was upregulated during osteogenic differentiation. ${ }^{* *} \mathrm{P}<0.01$, compared with the control group. (C) Western blotting results suggested that DHRS3 expression increased after induction of osteogenic differentiation. ${ }^{* *} \mathrm{P}<0.01$, compared with control group.

successful plasmid transfection (Fig. $6 \mathrm{~B}, P<0.01)$. The results of both qRTPCR and western blotting showed that the expression of DHRS3 was significantly downregulated with miR-223 overexpression, whereas the inhibition of miR-223 markedly elevated the expression of DHRS3 (Fig. 6C-D, $P<0.01$ ), indicating that miR-223 negatively regulates the expression of $D H R S 3$. Meanwhile, the expression of $D H R S 3$ was upregulated in the cDNA group, suggesting that the overexpression of DHRS3 (DHRS3 cDNA) was successfully obtained. The protein expression level of the cells was detected on day 14 after the induction of osteogenic differentiation. The ALP staining and ARS staining results showed that on day 14, ALP activity and calcification were enhanced in both the cDNA and antagomir groups, while the group simultaneously co-transfected with miR-223 agomir and DHRS3 cDNA showed no significant difference in ALP activity and calcification from the NC group (Fig. 7A-B, $P<0.01$ ). Consistent with the results above, the western blotting results showed that the expression levels of Runx2, OPN and OCN were upregulated in both the cDNA and antagomir groups, but there was no significant difference in protein expression in the group simultaneously co-transfected with miR-223 agomir and DHRS3 cDNA (Fig. 7 C, $P<0.01$ ). The above results demonstrated that miR-223 targeted DHRS3 and inhibited osteogenic differentiation.
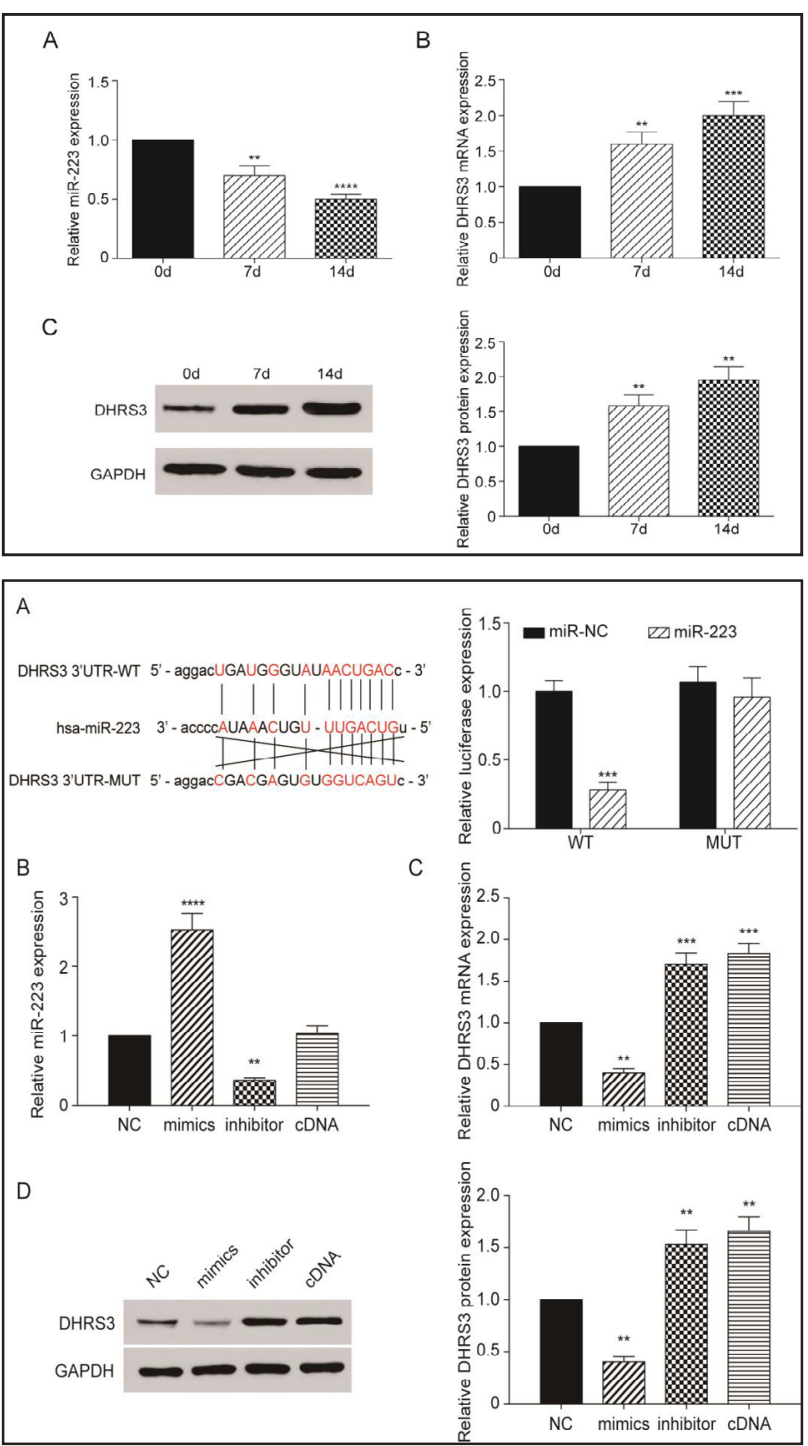

Fig. 6. MiR-223 targeted the 3'UTR region of DHRS3. (A) miR-223 targeted the DHRS3 3'UTR. Dual luciferase reporter gene assay results demonstrated that the luciferase activity in the miR-223 mimics+WT group was significantly weaker than in the $\mathrm{NC}+\mathrm{WT}$ group or the MUT recombinant plasmid groups. ${ }^{* * *} \mathrm{P}<0.001$, compared with the NC group. (A) QRTPCR results showed that the expression of miR-223 was increased in the agomir group, while it was decreased in the antagomir group. ${ }^{* *} \mathrm{P}<0.01$, compared with the NC group. (B-C) QRT-PCR and western blotting results detected that DHRS3 expression was significantly upregulated in both the DHRS3 cDNA and miR-223 antagomir groups, whereas it was downregulated in the miR-223 agomir group. ${ }^{* *} \mathrm{P}<0.01$, compared with the NC group. 


\section{Discussion}

Through microarray and enrichment analyses, DHRS3 was found to be differentially expressed and retinol metabolism was discovered to be activated during osteogenic differentiation. Through MiRanda and TargetScan, we found that DHRS3 may be targeted by miR-223. Our further studies showed that the expression of miR-223 decreased, while that of DHRS3 increased during osteogenic differentiation. In addition, osteogenic differentiation was upregulated after knocking down miR-223, while there were no obvious changes when miR-223 and DHRS3 were simultaneously overexpressed. The above results revealed that miR-223 suppressed retinol metabolism by directly targeting DHRS3, thereby inhibiting the osteogenic differentiation of hBMSCs.

Currently, Gene Set Enrichment Analysis (GSEA) has been widely used to analyze gene expression profiles. The goal of GSEA is to identify pathways where the constituent genes show coordinated changes in expression during the experimental conditions. GSEA is able to highlight genes connected to the phenotype through pathway analysis, even if the connection is weak [25].

Therefore, to clarify some potential genes and pathways involved in osteogenic differentiation, GSEA is likely to be more powerful than the conventional single-gene method. Through GSEA, we found that retinol metabolism was activated during osteogenic differentiation. Among the genes involved in retinol metabolism, DHRS3 was significantly upregulated.

DHRS3 is involved in the control of retinol metabolism and is codependent with retinol dehydrogenase 10 (RDH10). The first step of retinol metabolism is a reversible step, in which retinol associates with the $D H R S 3 / R D H 10$ complex and is oxidized to retinaldehyde by RDH10, which process is enabled by $D H R S 3$. Retinaldehyde can then be reduced back to retinol by the RDH10-actvated DHRS3, or it can be further irreversibly oxidized to retinoic acid (RA) by the ALDH genes $[22,26]$. By functionally activating each other, DHRS3 and RDH10 work together to maintain the appropriate level of RA [23]. Many studies have demonstrated the significant role of RA in osteogenic differentiation. It was reported that RA promoted the osteogenic

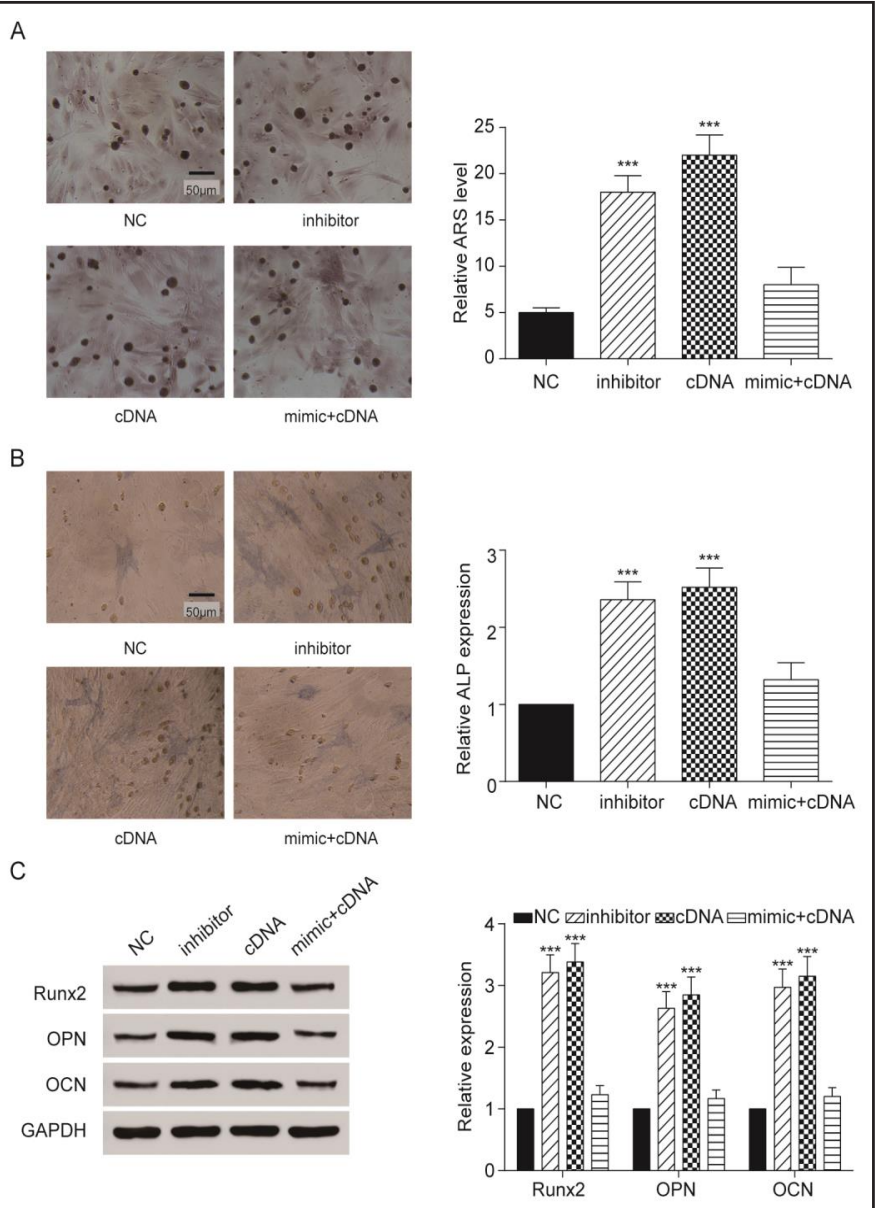

Fig. 7. MiR-223 inhibited osteogenic differentiation via targeting DHRS3. (A-B) ALP and ARS staining results demonstrated that inhibited miR-223 or overexpressed DHRS3 significantly increased the osteogenic differentiation of hBMSCs. The group simultaneously co-transfected with DHRS3 cDNA and miR-223 agomir showed no with the NC group. (C) The expression levels of Runx2, OPN and OCN were higher in the miR-223 antagomir group and the cDNA group than in the NC group. There was no significant difference between the NC group and the agomir+cDNA group. ${ }^{* * *} \mathrm{P}<0.001$, compared with the NC group. Scale bar: $50 \mu \mathrm{m}$. 
differentiation of mouse adipose-derived adult stromal cells (ADAS), suggesting that the osteogenic commitment of mouse ADAS requires RA [27]. In addition, other study found that BMP9-induced ALP activity, one of the early osteogenic markers, was significantly enhanced by RA in a dose-dependent manner. The results strongly suggested that RA may function synergistically with BMP9 activity to promote the osteogenic differentiation of MPCs, which could potentially be useful in bone regeneration therapy [28]. As has been mentioned above, in association with $R D H 10, D H R S 3$ plays a critical role in maintaining appropriate levels of RA, which promotes in a dose-dependent manner the osteogenic differentiation of MPCs. Consequently, there are strongly supported reasons to believe that DHRS3 plays a significant role during osteogenic differentiation. In the present study, we further found that DHRS3 can promote osteogenic differentiation in vitro.

Recent reports have demonstrated regulatory roles for miR-223 in bone metabolism, including in mediating the repression of the key proteins required for osteoblast differentiation [29]. For instance, miR-223 downregulates osteoblast differentiation through a C/EBP $\alpha / m i R-223 / F G F R 2$ regulatory feedback loop [29]. To maintain physiological bone metabolism, osteoblasts and osteoclasts coordinate with each other and maintain the balance between bone formation and resorption, which is always determined by the differentiation of osteoblasts and osteoclasts [30]. Once osteoblast differentiation decreases or osteoclast differentiation is enhanced, abnormal bone metabolism leads to a number of pathological bone destruction diseases [31]. It was reported that regulatory networks involving miR-223 play multiple roles in regulating bone metabolism. MiR-223 is a critical regulator in diseases with bone disorders, and changes in its expression are associated with the development stages and the control of clinical signs [17]. In the present study, we found that the expression of miR-223 gradually decreased during osteogenic differentiation, while the expression of DHRS3 gradually increased. By directly targeting DHRS3, rather than Runx2, OCN, or OPN, miR-223 could inhibit osteogenic differentiation.

There were some limitations in this study. First, through GSEA, several pathways were screened out, which might also be involved in osteogenic differentiation. However, because of limited time, we did not study the pathways and genes other than retinol metabolism and DHRS3. Second, only in vitro experiments were performed due to limited funding, and it is necessary to perform in vivo experiments to confirm the effect of retinol metabolites on osteogenic differentiation. Third, there are studies indicating that excess retinol stimulates bone resorption and inhibits bone formation, which might contribute to bone loss and further lead to osteoporosis [32]. Therefore, more experiments are needed to clarify the effect of different doses of retinol on bone formation and absorption. Finally, in the present study, we demonstrated that the overexpression of miR-223 inhibited the osteogenic differentiation of hBMSCs, but the effect of miR-223 on osteoblastic proliferation after $14 \mathrm{~d}$ requires further investigation.

\section{Conclusion}

In conclusion, we found that miR-223 and DHRS3 play significant roles during osteogenic differentiation. MiR-223 inhibited osteogenic differentiation via targeting DHRS3, providing the basis for future research.

\section{Acknowledgements}

This work was supported by grants from the National Natural Science Foundation of China (NO. 81701008); Natural Science Foundation of Shandong Province (NO. BS2015YY027); China Postdoctoral Science Foundation (N0.2014M561941); Special funds for Postdoctoral Innovation Projects of Shandong Province (N0.201402030); the Fundamental Research Funds of Shandong University (NO.201699000070) and the Horizontal Scientific Research Project of Shandong University (N0.12671726).

\section{KARGER}




\section{Cellular Physiology Cell Physiol Biochem 2018;47:667-679

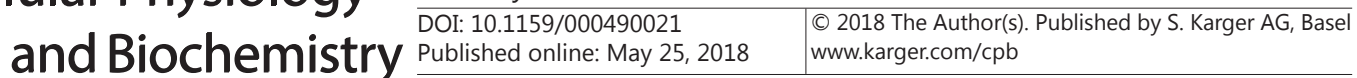 Zhang et al.: MiR-223/DHRS3 and Osteoblast Differentiation}

\section{Disclosure Statement}

No conflict of interests exists.

\section{References}

$\rightarrow 1$ Watson L, Elliman SJ, Coleman CM: From isolation to implantation: a concise review of mesenchymal stem cell therapy in bone fracture repair. Stem Cell Res Ther 2014;5:51.

-2 See EY, Toh SL, Goh JC: Multilineage potential of bone-marrow-derived mesenchymal stem cell cell sheets: implications for tissue engineering. Tissue Eng Part A 2010;16:1421-1431.

-3 Wakitani S, Saito T, Caplan AI: Myogenic cells derived from rat bone marrow mesenchymal stem cells exposed to 5-azacytidine. Muscle Nerve 1995;18:1417-1426.

4 Haynesworth SE, Goshima J, Goldberg VM, Caplan AI: Characterization of cells with osteogenic potential from human marrow. Bone 1992;13:81-88.

5 Friedenstein AJ, Petrakova KV, Kurolesova AI, Frolova GP: Heterotopic of bone marrow. Analysis of precursor cells for osteogenic and hematopoietic tissues. Transplantation 1968;6:230-247.

6 Zhang YD, Zhao SC, Zhu ZS, Wang YF, Liu JX, Zhang ZC, Xue F: Cx43- and Smad-Mediated TGF-beta/ BMP Signaling Pathway Promotes Cartilage Differentiation of Bone Marrow Mesenchymal Stem Cells and Inhibits Osteoblast Differentiation. Cell Physiol Biochem 2017;42:1277-1293.

-7 Zhou W, Yu L, Fan J, Wan B, Jiang T, Yin J, Huang Y, Li Q, Yin G, Hu Z: Endogenous Parathyroid Hormone Promotes Fracture Healing by Increasing Expression of BMPR2 through cAMP/PKA/CREB Pathway in Mice. Cell Physiol Biochem 2017;42:551-563.

-8 Fang S, Deng Y, Gu P, Fan X: MicroRNAs regulate bone development and regeneration. Int J Mol Sci 2015;16:8227-8253.

-9 Wei J, Shi Y, Zheng L, Zhou B, Inose H, Wang J, Guo XE, Grosschedl R, Karsenty G: miR-34s inhibit osteoblast proliferation and differentiation in the mouse by targeting SATB2. J Cell Biol 2012;197:509-521.

10 Huang J, Meng Y, Liu Y, Chen Y, Yang H, Chen D, Shi J, Guo Y: MicroRNA-320a Regulates the Osteogenic Differentiation of Human Bone Marrow-Derived Mesenchymal Stem Cells by Targeting HOXA10. Cell Physiol Biochem 2016;38:40-48.

11 Li G, Luo W, Abdalla BA, Ouyang H, Yu J, Hu F, Nie Q, Zhang X: miRNA-223 upregulated by MYOD inhibits myoblast proliferation by repressing IGF2 and facilitates myoblast differentiation by inhibiting ZEB1. Cell Death Dis 2017;8:e3094.

12 Lu H, Buchan RJ, Cook SA: MicroRNA-223 regulates Glut4 expression and cardiomyocyte glucose metabolism. Cardiovasc Res 2010;86:410-420.

-13 Zardo G, Ciolfi A, Vian L, Starnes LM, Billi M, Racanicchi S, Maresca C, Fazi F, Travaglini L, Noguera N, Mancini M, Nanni M, Cimino G, Lo-Coco F, Grignani F, Nervi C: Polycombs and microRNA-223 regulate human granulopoiesis by transcriptional control of target gene expression. Blood 2012;119:4034-4046.

-14 Taipaleenmaki H, Bjerre Hokland L, Chen L, Kauppinen S, Kassem M: Mechanisms in endocrinology: microRNAs: targets for enhancing osteoblast differentiation and bone formation. Eur J Endocrinol 2012;166:359371.

15 Xu J, Yao Q, Hou Y, Xu M, Liu S, Yang L, Zhang L, Xu H: MiR-223/Ect2/p21 signaling regulates osteosarcoma cell cycle progression and proliferation. Biomed Pharmacother 2013;67:381-386.

16 Shibuya H, Nakasa T, Adachi N, Nagata Y, Ishikawa M, Deie M, Suzuki O, Ochi M: Overexpression of microRNA-223 in rheumatoid arthritis synovium controls osteoclast differentiation. Mod Rheumatol 2013;23:674-685.

17 Xie Y, Zhang L, Gao Y, Ge W, Tang P: The Multiple Roles of Microrna-223 in Regulating Bone Metabolism. Molecules 2015;20:19433-19448.

18 Brossaud J, Pallet V, Corcuff JB: Vitamin A, endocrine tissues and hormones: interplay and interactions. Endocr Connect 2017. 


\section{Cellular Physiology Cell Physiol Biochem 2018;47:667-679 \begin{tabular}{l|l} 
DOI: 10.1159/000490021 & Ond 2018 The Author(s). Published by S. Karger AG, Basel \\
www.karger.com/cpb
\end{tabular} \\ Zhang et al.: MiR-223/DHRS3 and Osteoblast Differentiation}

19 Skillington J, Choy L, Derynck R: Bone morphogenetic protein and retinoic acid signaling cooperate to induce osteoblast differentiation of preadipocytes. J Cell Biol 2002;159:135-146.

-20 Yang QJ, Zhou LY, Mu YQ, Zhou QX, Luo JY, Cheng L, Deng ZL, He TC, Haydon RC, He BC: All-trans retinoic acid inhibits tumor growth of human osteosarcoma by activating Smad signaling-induced osteogenic differentiation. Int J Oncol 2012;41:153-160.

-21 Deisenroth C, Itahana Y, Tollini L, Jin A, Zhang Y: p53-Inducible DHRS3 is an endoplasmic reticulum protein associated with lipid droplet accumulation. J Biol Chem 2011;286:28343-28356.

-22 Shannon SR, Moise AR, Trainor PA: New insights and changing paradigms in the regulation of vitamin A metabolism in development. Wiley Interdiscip Rev Dev Biol 2017;6.

-23 Metzler MA, Sandell LL: Enzymatic Metabolism of Vitamin A in Developing Vertebrate Embryos. Nutrients 2016;8.

24 Billings SE, Pierzchalski K, Butler Tjaden NE, Pang XY, Trainor PA, Kane MA, Moise AR: The retinaldehyde reductase DHRS3 is essential for preventing the formation of excess retinoic acid during embryonic development. FASEB J 2013;27:4877-4889.

25 He K, Chen Z, Ma Y, Pan Y: Identification of high-copper-responsive target pathways in Atp7b knockout mouse liver by GSEA on microarray data sets. Mamm Genome 2011;22:703-713.

26 Adams MK, Belyaeva OV, Wu L, Kedishvili NY: The retinaldehyde reductase activity of DHRS3 is reciprocally activated by retinol dehydrogenase 10 to control retinoid homeostasis. J Biol Chem 2014;289:1486814880.

27 Wan DC, Shi YY, Nacamuli RP, Quarto N, Lyons KM, Longaker MT: Osteogenic differentiation of mouse adipose-derived adult stromal cells requires retinoic acid and bone morphogenetic protein receptor type IB signaling. Proc Natl Acad Sci U S A 2006;103:12335-12340.

28 Zhang W, Deng ZL, Chen L, Zuo GW, Luo Q Shi Q, Zhang BQ, Wagner ER, Rastegar F, Kim SH, Jiang W, Shen J, Huang E, Gao Y, Gao JL, Zhou JZ, Luo J, Huang J, Luo X, Bi Y, Su Y, Yang K, Liu H, Luu HH, Haydon RC, He TC, He BC: Retinoic acids potentiate BMP9-induced osteogenic differentiation of mesenchymal progenitor cells. PLoS One 2010;5:e11917.

29 Guan X, Gao Y, Zhou J, Wang J, Zheng F, Guo F, Chang A, Li X, Wang B: miR-223 Regulates Adipogenic and Osteogenic Differentiation of Mesenchymal Stem Cells Through a C/EBPs/miR-223/FGFR2 Regulatory Feedback Loop. Stem Cells 2015;33:1589-1600.

-30 Tamma R, Zallone A: Osteoblast and osteoclast crosstalks: from OAF to Ephrin. Inflamm Allergy Drug Targets 2012;11:196-200.

-31 Sucur A, Katavic V, Kelava T, Jajic Z, Kovacic N, Grcevic D: Induction of osteoclast progenitors in inflammatory conditions: key to bone destruction in arthritis. Int Orthop 2014;38:1893-1903.

-32 Binkley N, Krueger D: Hypervitaminosis A and bone. Nutr Rev 2000;58:138-144. 